\title{
EFEITO DE UM PROGRAMA DE FISIOTERAPIA AQUÁTICA NO EQUILÍBRIO E CAPACIDADE FUNCIONAL DE IDOSOS
}

\section{Alisson Felipe Siqueira}

Discente do curso de Fisioterapia - Faculdade Guairacá, Brasil.

\section{Denise Barth Rebesco}

Mestranda do Programa de Pós-graduação Interdisciplinar em Desenvolvimento Comunitário (UNICENTRO), Brasil.

\section{E-mail: deniserebesco@gmail.com}

\section{Franciele Aparecida Amaral}

Docente do curso de Fisioterapia - Faculdade Guairacá, Brasil.

\section{Claudia Bernardes Maganhini}

Docente do curso de Fisioterapia - Faculdade Guairacá, Brasil.

\section{Simone Mader Dall Agnol}

Mestranda do Programa de Pós-graduação Interdisciplinar em Desenvolvimento Comunitário (UNICENTRO), Brasil.

\section{Meiriélly Furmann}

Mestranda do Programa de Pós-Graduação Interdisciplinar em Desenvolvimento Comunitário (UNICENTRO), Brasil.

\section{Luis Paulo Gomes Mascarenhas}

Doutor Docente e Coordenador do Programa de Pósgraduação Interdisciplinar em Desenvolvimento Comunitário (UNICENTRO), Brasil.
RESUMO: O objetivo do estudo foi avaliar o efeito de um programa de fisioterapia aquática para o equilíbrio e capacidade funcional de idosos. Trata-se de um estudo clínico, prospectivo e de delineamento longitudinal com idosos cadastrados em um grupo de hipertensos e diabéticos $(n=11)$. O equilíbrio foi avaliado por meio do teste de Tinetti e a capacidade funcional foi avaliada através do teste de caminhada de 6 minutos. A intervenção consistiu de 3 sessões semanais de fisioterapia aquática com duração de 40 minutos cada, por um período de dois meses. Foram realizados testes pré e pós-intervenção, com inclusão de grupo-controle. A análise estatística foi realizada com auxílio do software SPSS-16. As variáveis quantitativas foram avaliadas pelo teste de normalidade Kolmogorov-Smirnov. Diante da normalidade, foi utilizado o teste T pareado com nível de significância de 0,05 . $O$ grupo experimental apresentou diferenças significativas $(p<0,05)$ no teste de equilíbrio, enquanto no teste que avaliou a capacidade funcional não houve diferença estatisticamente significativa $(p>0.05)$. Entretanto, observou-se melhora nos resultados pós-intervenção. $\mathrm{O}$ grupo estudado não apresentou melhoras significativas com relação à capacidade funcional, porém a fisioterapia aquática melhorou estatisticamente o equilíbrio dos idosos participantes. Neste sentido, a hidroterapia pode ser incluída em programas de promoção à saúde e prevenção de doenças, minimizando o impacto do processo de envelhecimento.

PALAVRAS-CHAVE: Senescência; Hidroterapia; Qualidade de vida; Equilíbrio postural; Promoção da saúde.

\section{EFFECT OF AN AQUATIC PHYSIOTHERAPY PROGRAM ON BALANCE AND FUNCTIONAL CAPACITY IN ELDERLY}

ABSTRACT: This study aims to assess the effect of aquatic physiotherapy for balance and functional capacity of elderly. This is a clinical, prospective, longitudinal study with elderly people enrolled in a group of hypertensive and diabetic patients $(n=11)$. The balance was assessed using the Tinetti test and the functional capacity through the 6-minute walk test. The intervention consisted of 3 weekly sessions of 40 minutes each of aquatic physiotherapy during 2 months. Pre and post intervention tests were performed, including with a control group. The statistical analysis was performed using SPSS-16 software. The quantitative variables were assessed by the Kolmogorov-Smirnov test. Face the normality, the paired T-test was utilized with a significance level of 0.05 The experimental group presented significant differences $(p<0.05)$ in the balance test, while there was no statistically significant difference in the functional capacity test $(p>0.05)$. However, it was noted an improvement in the post-intervention results. The group 
studied did not present significant improvements in regarding the functional capacity, but the aquatic physiotherapy statistically improved the balance of the elderly who participated of the study. In this regard, the hydrotherapy can be included in health promotion and disease prevention programs, minimizing the impact of the aging process.

KEY WORDS: Senescence; Hydrotherapy; Quality of Life; Postural Balance; Health Promotion.

\section{INTRODUÇÃO}

O envelhecimento tem sido descrito como um processo inerente a todos os seres vivos e que se caracteriza pela perda da capacidade de adaptação ao ambiente e pela redução da funcionalidade. O mesmo é conceituado como um processo dinâmico e progressivo, no qual ocorrem modificações morfológicas, funcionais, bioquímicas e psicológicas que determinam a perda da capacidade de adaptação do indivíduo ao meio ambiente, ocasionando, desta forma, maior vulnerabilidade e maior incidência de processos patológicos (COELHOet al, 2014).

Dentre as diversas alterações que ocorrem no processo de envelhecimento, os distúrbios relacionados ao equilíbrio estão entre os mais alarmantes devido ao alto índice de quedas, o que pode gerar perda de autonomia e independência para a realização de atividades básicas da vida diária (MARTINS; DASCAL; MARQUES, 2013). Pesquisa realizada por Oliveira et al (2014) evidenciou que mais da metade das quedas em idosos, ou seja, 55\%, ocorre em ambiente externo ao domicílio, sendo jardins, pátios, calçadas e ruas os locais de maior ocorrência.

As alterações do equilíbrio corporal estão entre as queixas mais comuns da população idosa e constituem um problema médico de grande relevância. Estima-se que a prevalência de queixas relacionadas ao equilíbrio chegue a $85 \%$ da população acima de 65 anos (HELRIGLEet al, 2013).

A capacidade funcional ou limitação funcional pode ser definida como a capacidade do indivíduo de cuidar de si próprio e viver de forma independente, ou seja, manter suas capacidades físicas e mentais em suas atividades básicas e instrumentais (KAGAWA;
CORRENTE, 2015). Ela vem sendo apontada como um importante indicador da saúde do idoso, tendo em vista que a presença de fatores limitantes, como doenças ou imobilidade, pode desencadear diferentes impactos na sua vida diária (SILVA e MENEZES, 2013).

A atividade física para o idoso é capaz de proporcionar efeitos orgânicos benéficos, incluindo o bem-estar geral, a preservação da independência, a prevenção e o tratamento de doenças e a diminuição de dores crônicas. Os programas de fisioterapia aquática têm sido frequentemente indicados como uma modalidade de atividade física para a população idosa em razão do ambiente ser seguro, menos sujeito a quedas e ter boa aceitação e adesão ao tratamento (SARMENTO, PEGORARO, CORDEIRO, 2011).

Estudos como os de Alcalde et al (2016), De Oliveira et al (2014) e Gimenes et al (2015) afirmam que exercícios em meio aquático são capazes de auxiliar idosos com diminuição da capacidade funcional, déficits de equilíbrio e limitações físicas inerentes ao avanço da idade. Sendo assim, o presente trabalho visou avaliar o efeito de um programa de fisioterapia aquática no equilíbrio e capacidade funcional de idosos.

\section{METODOLOGIA}

Trata-se de um estudo clínico, prospectivo e de delineamento longitudinal, realizado em uma clínica cscola localizada no município de Guarapuava, Paraná.

A pesquisa foi aprovada pelo Comitê de Ética e Pesquisa em Seres Humanos da Universidade Estadual do Centro Oeste, Guarapuava-PR, sob o Parecer $\mathrm{n}^{\circ}$ 1.142.297, e pela Resolução $n^{\circ} 466 / 12$, do Conselho Nacional de Saúde/CNS.

O convite para participar da pesquisa foi feito a idosos cadastrados em um grupo de hipertensos e diabéticos do programaHIPERDIAda $5{ }^{\text {a }}$ Regional de Saúde. Os critérios de inclusão e exclusão foram estabelecidos através de um questionário autoadministrado que estabelecia como critério de inclusão conseguir realizar marcha independente e realizar suas atividades de vida diária sem ajuda, apresentar pleno entendimento do processo da pesquisa, assinar o termo de consentimento 
livre e esclarecido e ter idade entre a 60 e 73 anos. Foram excluídos participantes sem marcha independente, que necessitassem de ajuda nas atividades de vida diária e idosos que estivessem praticando outro tipo de atividade física regularmente.

Para caracterizar o perfil dos idosos, foram analisados alguns dados pessoais, como peso, estatura e escolaridade.

Os testes para avaliação do equilíbrio e capacidade funcional foram aplicados pelo mesmo pesquisador, sendo que cada participante foi orientado individualmente sobre eles.

O Teste de Tinetti (SHUMWAY-COOK, 1997) foi usado para avaliar o equilíbrio e as anormalidades da marcha. O mesmo consiste de 16 itens, sendo que 9 são sobre o equilíbrio do corpo e 7 de marcha. O Teste de Tinetti classifica os aspectos da marcha, como velocidade, distância do passo, simetria, equilíbrio em pé, girar e as mudanças com os olhos fechados. A contagem para cada exercício varia de 0 a 1 ou de 0 a 2 , com uma contagem mais baixa que indica uma habilidade física mais pobre. A pontuação total é a soma da pontuação do equilíbrio do corpo e a da marcha. A pontuação máxima é 12 pontos para a marcha, 16 pontos para o equilíbrio do corpo e 28 pontos totais (SHUMWAY-COOK, 1997).

$\mathrm{O}$ teste foi aplicado com o indivíduo sentado em uma cadeira sem apoio de braços para o exame do equilíbrio sentado. Para análise da marcha, observou-se a caminhada do participante em um corredor andando em velocidade normal, voltando ao ponto de origem com passos rápidos, porém seguros.A pontuação total foi a soma da pontuação do equilíbrio do corpo e da marcha, sendo que a pontuação total do índice é 28 pontos e pontuações menores que 19 indicam risco cinco vezes maior de quedas (Soares et al, 2012).

Em seguida foi realizada a avaliação da capacidade funcional. Para esta, foi feita um teste de caminhada de 6 minutos (TC6) que é usado para avaliar a capacidade física e a capacidade submáxima do exercício. Trata-se de uma intervenção simples, segura e bem tolerada pelos pacientes (DE ASSIS RAMOS e DE SÁ FERREIRA, 2014).

O teste foi realizado em um corredor de 30 metros de comprimento, demarcado de 3 em 3 metros, sendo que o momento de fazer a curva para a volta foi marcado com um cone. Nesse percurso, o idoso caminhou até os cones sem falar com as pessoas ao seu redor e voltou rapidamente em torno deles, continuando a caminhada sem hesitação (JÚNIOR et al, 2015). O caminho percorrido foi mostrado previamente ao idoso pelo examinador.

A velocidade da caminhada foi determinada pelos próprios participantes, enquanto um dos avaliadores os acompanhava durante todo o percurso do teste. Para evitar a indução do ritmo de caminhada pelo avaliador, este posicionou-se atrás do idoso, dandolhe uma das mãos, quando necessário (AMERICAN THORACIC SOCIETY, 2002). A frequência cardíaca (FC) foi monitorada continuamente durante os testes e o receptor ficava com o avaliador que acompanhava o idoso durante todo o percurso. A pressão arterial (PA) foi mensurada com um esfigmomanômetro aneroide (fase I e V dos sons de Korotkoff) e a frequência respiratória (FR) foi determinada por meio da contagem do número de incursões respiratórias por minuto, decorrentes do movimento do gradil costal dos participantes. Antes do teste (após cinco minutos de repouso sentado) e ao seu final foram aferidos manualmente a FC, FR e a PA. A saturação periférica de oxigênio foi verificada com um oxímetro de pulso de dedo da marca Moriya e o grau de esforço percebido foi verificado pela escala de Borg modificada (BORG, 2000).

A intervenção da fisioterapia aquática foi realizada de agosto a outubro de 2016 com uma frequência de três vezes por semana, às segundas, quartas e sextas-feiras, com cada sessão tendo duração de 40 minutos. Os exercícios aquáticos foram feitos em uma piscina com 6,66 metros de comprimento, 3,80 metros de largura e 1,20 metros de profundidade, e água à temperatura de $34^{\circ} \mathrm{C}$. Segundo Oliveira et al (2014), a temperatura ideal para atividades aquáticas está entre 33 e $36^{\circ} \mathrm{C}$. Para a aplicação do protocolo, o pesquisador permaneceu na borda da piscina, sendo responsável por mostrar o exercício a ser executado e controlar o tempo e as repetições. Três outros instrutores ajudaram no posicionamento e execução correta dos exercícios dentro da piscina, além de evitarem possíveis quedas e acidentes. Houve um período de descanso de dez segundos entre cada exercício. Nos exercícios aeróbicos 
e de fortalecimento, os participantes foram orientados a manter uma frequência de 40 a 60 repetições por minuto.

Cada sessão foi composta por exercícios de aquecimento, fortalecimento de membros superiores e membros inferiores, equilíbrio com os olhos abertos e fechados, exercícios aeróbicos e alongamento. Após o término dos dois meses do programa de tratamento, os participantes foram reavaliados para obtenção de resultados pós-intervenção.

A análise estatística foi realizada com o software SPSS-16. As variáveis quantitativas foram avaliadas pelo teste de normalidade Kolmogorov-Smirnov. Diante da normalidade, foi utilizado o teste $\mathrm{T}$ pareado com nível de significância de 0,05 . As variáveis qualitativas foram expressas em frequência e porcentagem.

\section{RESULTADOS}

A amostra é composta por 11 indivíduos com idades entre 60 e 73 anos $(66,55 \pm 4,34)$ e IMC entre 25,90 e $37,25(30,68 \pm 3,78)$.

A maioria dos indivíduos analisados era do sexo feminino $(81,82 \%)$, sendo somente dois indivíduos do sexo masculino $(18,18 \%)$. Foram encontradas diferenças significativas $(\mathrm{p}<0,05)$ entre os resultados pré e pósintervenção no teste de Tinetti, com média e desvio padrão de 20,73 $\pm 3,43$ na pré-intervenção, aumentando para 26,73 $\pm 1,00$ na pós-intervenção. No TC6 não houve diferença estatisticamente significativa $(p>0.05)$, porém houve um aumento nos resultados pós-intervenção, sendo 430,08 $\pm 98,70$ no pré-intervenção e 438,18 $\pm 13,17$ no pós- intervenção (Tabela 1).

Tabela 1. Comparação entre as variáveis Tinetti e TC6 min nos momentos de pré e pós-intervenção

\begin{tabular}{|c|c|c|c|}
\hline \multirow[b]{2}{*}{ Testes } & Pré & Pós & \multirow[b]{2}{*}{$P$} \\
\hline & $\begin{array}{c}\text { Média e } \\
\text { Desvio Padrão }\end{array}$ & $\begin{array}{c}\text { Média e } \\
\text { Desvio Padrão }\end{array}$ & \\
\hline Tinetti & $20,73 \pm 3,43$ & $26,73 \pm 1,00$ & $0,000 *$ \\
\hline TC6min & $430,08 \pm 98,70$ & $438,18 \pm 13,17$ & 0,675 \\
\hline
\end{tabular}

Fonte: Dados da pesquisa

Nos resultados pré-intervenção do teste de Tinetti, 3 participantes $(27,3 \%)$ foram classificados com alto risco de quedas, 6 participantes $(54,5 \%)$ com risco moderado de quedas e 2 participantes $(18,2 \%)$ com baixo risco de quedas. Nos resultados pós-intervenção, os 11 participantes (100\%) foram classificados com baixo risco de quedas. Assim, os resultados indicam que o grupo estudado teve aumento estatisticamente significativo $(p<0,05)$ em relação ao equilíbrio após a intervenção aquática. Esses dados podem ser observados na Tabela 2.

\section{Tabela 2: Classificação do teste Tinetti}

Frequência e porcentagem do teste Tinetti de acordo com a classificação

\begin{tabular}{cccc}
\hline Tinetti & $\begin{array}{c}\text { Baixo risco } \\
\text { N - \% }\end{array}$ & $\begin{array}{c}\text { Moderado risco } \\
\text { N - \% }\end{array}$ & $\begin{array}{c}\text { Alto risco } \\
\text { N - \% }\end{array}$ \\
\hline Pré-intervenção & $2-18,2 \%$ & $6-54,5 \%$ & $3-27,3 \%$ \\
\hline Pós-intervenção & $11-100 \%$ & - & - \\
\hline
\end{tabular}

Fonte: Dados da pesquisa

$O$ teste de caminhada de 6 minutos não apresentou resultados estatisticamente significativos ( $p>0.05$ ): a média na pré-intervenção foi $430,08 \pm 98,70$, sendo esperado o resultado de $442,73 \pm 23,00$, de acordo com a faixa etária. O resultado pós-intervenção foi 438,18 $\pm 43,70$ estando ainda abaixo do esperado. Mesmo não havendo resultados significativos, houve um aumento de 8,10 metros nas médias pré e pós-intervenção. Os resultados do TC6 estão apresentes na Tabela 3.

Tabela 3. Comparação das médias pré e pós com a média esperada do TC6 min

Valores de média e desvio-padrão dos valores pré, pósintervenção e esperado do TC6 min

\begin{tabular}{cccccc}
\hline & $\mathbf{N}$ & Pré & Pós & Esperado & $\boldsymbol{p}$ \\
\hline \multirow{2}{*}{ TC6min } & 11 & $430,08 \pm 98,70$ & - & $442,73 \pm 23,00$ & 0,639 \\
\cline { 2 - 6 } & \cline { 2 - 6 } & - & $438,18 \pm 43,70$ & $442,73 \pm 23,00$ & 0,681 \\
\cline { 2 - 6 }
\end{tabular}

\section{DISCUSSÃO}

Estudos, como os de Chang, et al (2017) Hulya, et al (2015), Kritchevsky, et al (2017),Tew, et al (2017),Liao, et al (2017), consideram a prática regular de atividade física, mesmo quando iniciada após os 65 anos, como algo que favorece a maior longevidade, a redução 
das taxas gerais de mortalidade, a prevenção do declínio cognitivo, a manutenção do status funcional, a redução da frequência de quedas, bem como a incidência de fraturas, além dos benefícios psicológicos e a melhoria da autoestima.

O presente estudo demonstrou que um programa de fisioterapia aquática pode melhorar o equilíbrio em idosos, minimizando os fatores de risco das quedas. Esses resultados corroboram com estudo feito por Bruni, Ganado e Prado (2008), que avaliou, por meio do teste de Tinetti, a influência da água no equilíbrio postural de 23 idosas divididas em 2 grupos (grupo inserido em programa de exercícios e grupo que recebeu orientações sobre prevenção de quedas, mas não fez exercícios). Os resultados apresentaram melhoria significativa para o grupo que realizou hidroterapia tanto no equilíbrio postural quanto na marcha.

É importante ressaltar que o envelhecimento populacional e o aumento da expectativa de vida demandam ações preventivas que possam controlar os fatores de risco das quedas e promovam medidas que visem a participação dessa população na prática de atividades físicas (SILVA et al,2008).

O objetivo desse estudo foi verificar a influência da fisioterapia aquática na capacidade funcional e no equilíbrio em idosos. O TC6 não apresentou resultado estatisticamente significativo com relação à capacidade funcional. No entanto, a participação em programas de exercícios, mesmo os de intensidade leve tem demonstrado ser útil para a redução significativa do número de quedas, quando compara-se os grupos controle que não realizam exercícios com os que os realizam (GUIMARÃES et al,2004; BENTO et al, 2010). O TC6 não ter alcançado significância na diferença dos valores pré e pós-intervenção possivelmente ocorreu em função de uma das limitações deste estudo, o seu tamanho amostral, que é pequeno demais para que se possa fazer inferências para a população.

Por outro lado, houve resultados estatisticamente significativos $(p<0,05)$ no teste de Tinetti, pois $100 \%$ da amostra foi classificada com baixo risco de quedas após a intervenção. Com isso, evidenciou-se que a fisioterapia aquática causu uma propensão à melhoria no equilíbrio postural e, consequentemente, redução do risco de quedas em idosos.
O processo normal do envelhecimento caracteriza-se pela diminuição da capacidade funcional dos diversos órgãos e tecidos, aumentando o risco de doenças crônicas degenerativas que estão tornandose prevalentes em nosso país (BARDUZZI et al, 2013). Todavia, programas de exercícios, como o utilizado neste estudo, podem atenuar o impacto causado pelo envelhecimento.

De acordo com Santos e Santos (2010), o exercício físico em idosos, quando realizado adequadamente, pode ter inúmeros benefícios, dentre eles aumento na capacidade funcional, diminuição da incidência de infecções, melhora da saúde cardiovascular, aumento da massa muscular, melhora no aspecto psicológico, entre outros.

Foi realizado um estudo por Oliveira et al(2014) com 74 idosas, divididas em 3 grupos, quais sejam, grupo cama elástica $(n=23)$, grupo fisioterapia aquática $(n=28)$ e grupo solo $(n=23)$, que realizaram 24 sessões de exercícios de força, flexibilidade, equilíbrio estático e dinâmico. Foi utilizado para avaliação do equilíbrio uma plataforma bipodal e unipodal, antes e após as intervenções. Ao término das sessões, o grupo de exercícios aquáticos obteve resultados melhores em comparação aos outros grupos. Dessa forma, o exercício na água torna-se ainda mais interessante, pois além da segurança que proporciona aos praticantes, em algumas vezes é também mais eficiente.

Além disso, Buzelliet al (2016), sugere que o centro de equilíbrio e a oscilação do tronco são influenciados pelo ambiente aquático, juntamente com os efeitos da informação visual. Neste estudo, 10 participantes realizaram uma avaliação de equilíbrio na água e no solo, utilizando uma placa de força e sensores para mensurar a oscilação do corpo.

Então, o ambiente aquático pode ser um estímulo para melhorar o controle do equilíbrio. Douriset al (2003) relatam que o suporte oferecido pela água permite maior independência na manutenção de posturas, fazendo com que os idosos tenham menos medo de movimentar-se.

No estudo de Bergaminet al (2013), realizado com 59 idosos divididos em três grupos, grupo aquático, grupo solo e grupo controle, os autores avaliaram o equilíbrio estático e dinâmico através do teste Time 
Upand Go. O grupo aquático e o grupo solo realizaram a intervenção semanalmente durante 6 meses. Os resultados evidenciaram que o grupo aquático teve melhoria significativa em comparação ao grupo solo e ao grupo controle.

A melhoria no equilíbrio deve-se à atração molecular no meio líquido que, quando posta em movimento, gera uma resistência (viscosidade) que é responsável pelo suporte oferecido aos idosos durante a hidroterapia. Isto sugere uma melhora do equilíbrio dentro da água, o que reflete em uma melhora em solo (RUOTI et al, 2000).

O presente estudo evidencia a importância da prática de atividades físicas na manutenção da saúde do idoso. Sua contribuição consiste em chamar a atenção para a saúde dos idosos, hoje alvo das políticas públicas de saúde que se mostram impulsionadoras na melhoria da qualidade de vida destes.

Nessa perspectiva, compreende-se haver possibilidades de minimizar ou eliminar alguns problemas que comprometem a saúde dessa população por meio de ações que potencializem a participação dela em atividades comunitárias enquanto estratégia de promoção da saúde

Como limitação do estudo, destaca- se a dificuldade dos participantes, que demonstraram insegurança com o meio aquático nas primeiras sessões. Além disso, o número da amostra foi limitado devido ao tamanho da piscina.

\section{CONCLUSÃO}

O grupo estudado não apresentou melhorias significativas com relação à capacidade funcional, porém a fisioterapia aquática melhorou significativamente o equilíbrio dos idosos participantes. Sugere-se a implantação de iniciativas que estimulem a prática da fisioterapia aquática para indivíduos idosos, sendo incluídas em programas de promoção de saúde e prevenção de doenças com o propósito de manter e melhorar a saúde dessa população, minimizando o impacto do processo de envelhecimento.

\section{REFERÊNCIAS}

ALCALDE, G. E., GUIMARÃES, L. E., PIANNA, B., DE ALMEIDA, A. D., ARCA, E. A., LIMA, M. A. X. C., \& FIORELLI, A. Impacto do programa de fisioterapia aquática na mobilidade funcional de idosos da comunidade. Kairós. Revista da Faculdade de Ciências Humanas e Saúde. ISSN 2176-901X, v. 19, n. 4, p. 243-253, 2016.

AMERICAN THORACIC SOCIETY. Statement: guidelines for thesix-minute walk test. American JournalofRespiratoryandCriticalCare Medicine, New York, v. 166, n. 1, p. 111-117, 2002.

BENTO, P. C. B., RODACKI, A. L. F., HOMANN, D., \& LEITE, N. Exercícios físicos e redução de quedas em idosos: uma revisão sistemática. RevBrasCineantropom Desempenho Hum, v. 12, n. 6, p. 471-9, 2010.

BARDUZZI, G. D. O.; ROCHA JÚNIOR, P. R.; SOUZA NETO, J. C. D.; AVEIRO, M. C. Functionalcapacityofelderlywithosteoarthritiswhoundergonetoaquaticandlandphysicaltherapy. Fisioterapia em movimento, v. 26, n. 2, p. 349- 360, 2013.

BERGAMIN, M.; ERMOLAO, A.; TOLOMIO, S.; BERTON, L.; SERGI, G.; ZACCARIA, M. Water-versus land-basedexercise in elderlysubjects: effectsonphysical performance andbodycomposition. Clinicalinterventions in aging, $\mathrm{v}$. 8, p. 1109-1117, 2013.

Borg G. Escalas de Borg para a Dor e o Esforço Percebido. São Paulo: Manole; 2000.

BUZELLI, A. R.; ROUHANI, H.; MASANI, K.; VERRIER, M. C.; POPOVIC, M. R. The influenceoftheaquaticenvironmentonthecontrolof postural sway. Gait\&Posture, v. 51, p. 70-76, 2016.

BRUNI, B. M.; GRANADO, F. B.; PRADO, R. A. Avaliação do equilíbrio postural em idosos praticantes de hidroterapia em grupo. O Mundo da Saúde, v.32, n. 1, p.56-63, 2008.

CHANG, P. S., KNOBF, M. T., FUNK, M., \& OH, B. FeasibilityandAcceptabilityofQigongExercise in Community-DwellingOlderAdults in the United States. The JournalofAlternativeandComplementary Medicine, 2017. 
COELHO, B. S.; SOUZA, L. K.; BORTOLUZZI, R.; RONCADA, C.; TIGGEMANN, C. L.; DIAS, C. P. Comparação da força e capacidade funcional entre idosos praticantes de musculação, hidroginástica e não praticantes de exercícios físicos, Revista Brasileira de Geriatria e Gerontologia, Rio de Janeiro, v. 17, n. 3, p. 497-504,2014.

DE ASSIS RAMOS, RODRIGO; DE SÁ FERREIRA, ARTHUR. Capacidadefuncional de adultos com hipertensãoavaliadapelo teste de caminhada de seisminutos: revisãosistemática. Fisioterapia e Pesquisa, v. 21, n. 3, p. 257-263, 2014 .

DE OLIVEIRA, A. S., TREVIZAN, P. F., BESTETTI, M. L. T., $\&$ DE MELO, R. C.Fatoresambientais e risco de quedasemidosos: revisãosistemática. RevistaBrasileira de Geriatria e Gerontologia, v. 17, n. 3, p. 637-645, 2014.

DOURIS, P.; SOUTHARD, V.; VARGA, C.; SCHAUSS, W.; GENNARO, C.; REISS, A. The Effectof Land andAquaticExerciseon Balance Scores in OlderAdults. JournalofGeriatricPhysicalTherapy, v. 26, p. 3-6, 2003.

GIMENES, C., ARCA, E. A., PAULINO, M. A., NICOLAU, N. V., BUITONI, B., PONTES, T. P., ... \& BARRILE, S. R. Redução da pressão arterial e circunferência abdominal e melhora da Capacidade Funcional de idosas hipertensas submetidas a Programa de Fisioterapia Funcional. Kairós Gerontologia. Revista da Faculdade de Ciências Humanas e Saúde. ISSN 2176-901X, v. 18, n. 1, p. 77-92, 2015.

GUIMARÃES, L. H. C. T., GALDINO, D. C. A., MARTINS, F. L. M., VITORINO, D. F. M., PEREIRA, K. L., \& CARVALHO, E. M.. Comparação da propensão de quedas entre idosos que praticam atividade física e idosos sedentários. RevNeurocienc, v. 12, n. 2, p. 68-72, 2004.

HELRIGLE, C.; FERRI, L. P.; NETTA, C. P. O.; BELEM, J. B.; MALYSZ.T. Efeitos de diferentes modalidades de treinamento físico e do hábito de caminhar sobre o equilíbrio funcional em idosos. Fisioterapia e Movimento. Curitiba. v.26, p. 321-327,2013.

HULYA, T. D., SEVI, Y. S. S., SERAP, A., \& AYSE. Factorsaffectingthebenefitsof a six-monthsupervisedexercisepro- gramoncommunity-dwellingolderadults: interactionsamong age, gender, andparticipation. Journalofphysicaltherapyscience, v. 27, n. 5, p. 1421-1427, 2015.

JUNIOR, N. S. F.; NAKATA, C. H.; OLIVEITA, L. V. F.; CHIAPPA, G. R.; JÚNIOR, G. C. Evaluationofthebestenvironment for thesix-minute walk test. Fisioterapia e movimento,Curitiba, v. 28, n. 3, p. 429-436, Set. 2015.

KAGAWA, C. A.; CORRENTE, J. E. Análise da capacidade funcional em idosos do município de Avaré- SP: fatores associados. Revista Brasileira de Geriatria e Gerontologia.Rio de Janeiro. v.18, n.3, p. 577- 586, 2015.

KRITCHEVSKY, S. B., LOVATO, L., HANDING, E. P., BLAIR, S., BOTOSENEANU, A., GURALNIK, J. M., ... \& REJESKI, W. J..Exercise'seffectonmobilitydisability in olderadultswithandwithoutobesity: The LIFE studyrandomizedclinicaltrial. Obesity, v. 25, n. 7, p. 1199-1205, 2017.

LIAO, C. D., TSAUO, J. Y., LIN, L. F., HUANG, S. W., KU, J. W., CHOU, L. C., \& LIOU, T. H. Effectsofelasticresistanceexerciseonbodycompositionandphysicalcapacity in olderwomenwithsarcopenicobesity: A CONSORT-compliantprospectiverandomizedcontrolledtrial. Medicine, v. 96, n. 23, 2017.

MARTINS, R. M.; DASCAL, J. B.; MARQUES, I. Equilíbrio postural em idosos praticantes de hidroginástica e karatê. Revista Brasileira de Geriatria e Gerontologia. Rio de Janeiro, v.16, n. 1, p. 61- 69, 2013.

OLIVEIRA, M. R.; DA SILVA, R. A.; DASCAL, J. B.; TEIXEIRA, D. C. Effectofdifferenttypesofexerciseon postural balance in elderlywomen: A randomizedcontrolledtrial. Archivesofgerontolog yandgeriatrics, v. 59, n. 3, p.506514, 2014.

RUOTI R.; MORRIS D.; COLE, A. Reabilitação aquática. São Paulo: Manole; 2000.

SANTOS, VINÍCIUS CONEGLIAN; SANTOS, ANIELY CONEGLIAN. Exercício físico e seus efeitos sobre o sistema imune dos idosos. Saúde e Pesquisa, v. 3, n. 2, 2010.

SARMENTO, G. S.; PEGORARO, A. S. N.; CORDEIRO, R. C. Fisioterapia aquática como modalidade de tratamento em 
idosos não institucionalizados: uma revisão sistemática.

Einstein. São Paulo, v. 9, n.1, p. 84-89, 2011.

SHUMWAY-COOK A, BALDWIN M, POLISSAR NL, GRUBER W. Predictingtheprobability for falls in communitydwellingolderadults. PhysicalTherapy. v. 77, n 8, p. 8129, 1997.

SILVA, A. D., TUFIK, S., MELLO, M. T. D., PECCIN, M. S., COHEN, M., CASSILHAS, R. C., \& ALMEIDA, G. J.. Equilíbrio, coordenação e agilidade de idosos submetidos à prática de exercícios físicos resistidos. RevBrasMed Esporte, Niterói, v.14, n. 2, p. 88-93, Apr. 2008.

SILVA, N. A.; MENEZES, T. N. Capacidade funcional e sua associação com idade e sexo em uma população idosa. Revista Brasileira de Cineantropometria e Desempenho Humano. v.16, p. 359, 2013.

SOARES, LISANDRA DELFINO DE ALBUQUERQUE., DE ARRUDA CÂMARA, F., DE ARAÚJO, M. D. G. R., FALCÃO, T., SIQUEIRA, A. P., ... \& DE FARO, Z. P. Análise do desempenho motor associado ao estado nutricional de idosos cadastrados no Programa Saúde da Família, no município de Vitória de Santo Antão-PE. Ciênc. Saúde coletiva, Rio de Janeiro, v. 17, n. 5, p. 1297-1304, May 2012.

TEW, GARRY A., HOWSAM, J., HARDY, M., \& BISSEL, L.. Adapted yoga to improve physicalfunctionandhealth-relatedqualityoflife in physically-inactiveolderadults: a randomisedcontrolledpilottrial. BMC geriatrics, v. 17, n. 1, p. $131,2017$.

Recebido em: 30 de maio de 2017 Aceito em: 24 de agosto de 2017 